\title{
HIGH-POWER ELECTRON LINAC FOR IRRADIATION APPLICATIONS
}

\author{
K.I. Antipov, M.I. Ayzatsky, Yu.I. Akchurin, V.A. Gurin, V.N. Boriskin, V.I. Beloglasov, \\ E.Z. Biller, N.V. Demidov, A.N. Dovbnya, I.V. Khodak, A.I. Kosoy, V.A. Kushnir, \\ L.K. Myakushko, V.V. Mitrochenko, T.F. Nikitina, S.A. Perezhogin, G.D. Pugachev, \\ O.A. Repikhov, L.V. Reprintzev, V.A. Shendrik, D.L. Stepin, G.E. Tarasov, A.E. Tolstoy, \\ Yu.D. Tur, V.F. Zhiglo, V.L. Uvarov \\ NSC "Kharkov Institute of Physics \& Technology”, Ukraine \\ M.A. Krasnogolovets, Yu.Ya. Volkolupov \\ Kharkov Technical University for Radioelectronics, Ukraine
}

\begin{abstract}
The paper presents calculated performances and the electron linac arrangement that is under installation at NSC KIPT. The linac consists of two 1.2-meter long piece-wise homogeneous accelerating structures and the injector including a diode electron gun, a klystron type buncher and an accelerating cavity. The wave phase velocity in the structures is equal to the velocity of the light. Each accelerating structure is powered with the Russian produced S-band klystron KIU-12AM. The linac is equipped with the beam scanning system to extract the beam through an air-cooled foil. The simulation of electron motion in the accelerator was carried out using the PARMELA code. When each accelerating structure is supplied with RF power of $11 \mathrm{MW}$ and the current at the accelerator exit is $1 \mathrm{~A}$, beam energy will be up to $20 \mathrm{MeV}$. An average beam power is planned to be $20 \mathrm{~kW}$.
\end{abstract}

\section{INTRODUCTION}

Powerful linear electron accelerators with energy up to $20 \mathrm{MeV}$ can be applied for radiation technologies, for instance for the radionuclide production using $(\gamma, \mathrm{n})$ reactions [1]. Such performances as electron efficiency, the accelerator length, probability of accelerating structures and beamline to be damaged by a beam and the reliability of a whole facility are important for technological accelerators. Accelerator KUT [2], was selected as a prototype for designed accelerator on the basis of a long-term operational experience. The accelerator KUT consists of the injector and one accelerating section with variable geometry and constant phase velocity. The injector includes $25 \mathrm{kV}$ diode electron gun, klystron type buncher and accelerating cavity. Length of the accelerating section is $1.2 \mathrm{~m}$, oscillations mode is $\Theta=2 \pi / 3$ at frequency $2797.2 \mathrm{MHz}$. Electron energy at its exit with beam current $1 \mathrm{~A}$ can reach $10 \mathrm{MeV}$. Using of one more the same section gets the electron energy of $20 \mathrm{MeV}$.

\section{SIMULATIONS}

As the operating of the accelerator with long enough current pulse duration $(\sim 4 \mu \mathrm{s})$ is supposed, only steadystate mode was considered. The electron gun optics and beam parameters at the gun [3] exit were calculated using the EGUN code [4]. Longitudinal and transverse beam dynamics in the accelerator were simulated using the PARMELA code [5]. Spatial distribution of magnetic field in axial focusing magnetic elements of the accelerator (three lenses and short solenoid on the first section) and spatial distribution of electromagnetic fields in the buncher and accelerating cavity were computed using the SUPERFISH/POISSON code [6]. This code was also used for spatial harmonics computing in the piecewise uniform structure (11 harmonics were taken into account). The calculation of amplitude and phase distribution of the fundamental spatial harmonic in the non-uniform structure taking into account beam loading has been made using a self-consistent one-dimensional model [7] by interaction region dividing into arrange of uniform sites matching to each other on an operational frequency. Energy and bunch phase length at the structure input were calculated using results of the injector simulation. Results of the self-consistent problem calculation were used for the amplitude and phase of fundamental spatial harmonic evaluating in each cell of the travelling wave (TW) accelerating structures, as it is necessary for PARMELA simulations. For the correct space charge forces accounting the input beam was set as a bunch having the length of $5 \beta \lambda$, where $\beta$-initial normalised velocity of particles, $\lambda$-operating wavelength.

The simulation has been carried out in several steps. During the first step there was investigated beam parameters at the injector exit depending on the phase shift between the buncher and accelerating cavity and on field amplitude in accelerating cavity under different magnetic field configurations and positions of elements of the injector. Being based on simulation results the injector layout was optimised. The optimal values of phase shift and field amplitude was selected in the accelerating cavity proceeding from obtained results and accessible RF power of $1 \mathrm{MW}$.

In the next step the first accelerating section was added to the injector and the phase shift between them giving the maximum energy gain was computed. Then the second section was added and the same procedure was carried out for the phase shift between sections. Fig. 1 indicates the envelope of the beam containing $95 \%$ of 
particles along the accelerator with different phase shifts between sections. One can see that the section has RF focussing. The beam diameter is less than the minimum aperture of the section $(\varnothing=19.6 \mathrm{~mm})$ even the solenoid is turned off.

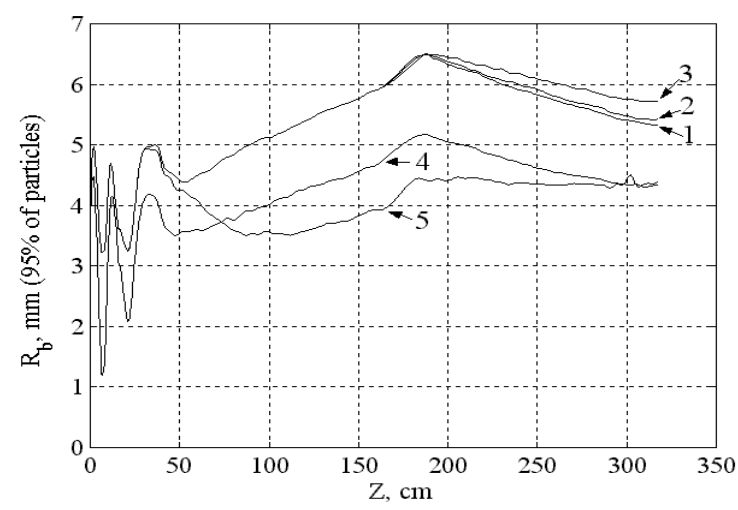

Figure 1: Beam enveloupe along the linac

1 - the solenoid turned off, bunches at the RF crest in the second section; 2 - the same case, but bunches at $18^{\circ}$ off the RF crest; 3 - the same case, but bunches at $36^{\circ}$ off the

RF crest; 4 - as the case 1 , but a space charge was not taken into account; 5 - as the case 1 , but the solenoid turned on $(B=500 \mathrm{G})$.

It is worthy of note that it is necessary to be careful choosing the solenoid field on the first section of such two-section accelerator because of the beam overfocusing can cause damage of elements of the accelerator target part. Fig. 2 indicates an average beam power dissipated along the accelerator (duty factor was equal $0.1 \%$ ). Such dissipated power does not represent a danger to the accelerator elements. The main part of particles $(26 \%)$ is lost in the injector, $10 \%$ of particles are lost in the first section and $1 \%$ is lost in the second section. Therewith the main energy loss falls at the first iris of the subsection with minimum iris size. The average power loosed in it will be $15 \mathrm{~W}$.

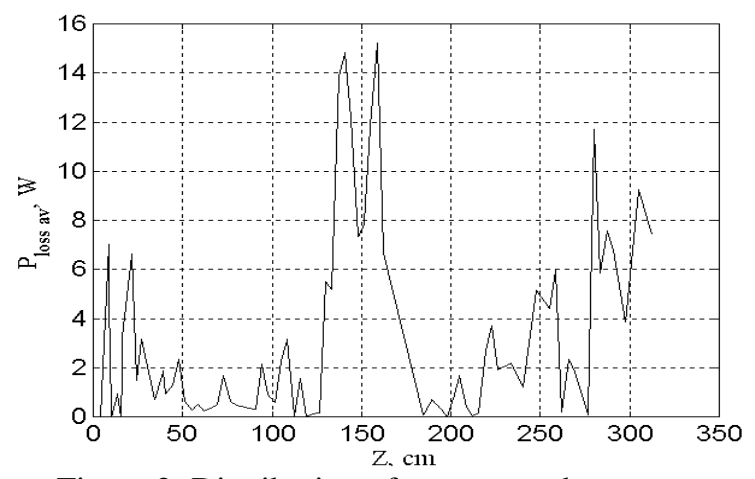

Figure 2: Distribution of an average beam power dissipated along the accelerator

The transverse beam profile analyzes at the accelerator exit showed the beam has a brightly expressed core. The beam FWHM is $2.5 \mathrm{~mm}$ (beam sizes for $70 \%$ and $90 \%$ of particles are indicated in table 1). Therewith the beam has halo limited by the aperture of the accelerator. The average power of $258 \mathrm{~W}(178 \mathrm{~W}$ in the first section with the injector and $82 \mathrm{~W}$ in the second section) is dissipated in the accelerator because of halo particle losing. It is about $1 \%$ of the average power of the accelerated beam. The beam parameters at the exit of sections are given in the table 1 .

Table 1

\begin{tabular}{|l|c|c|}
\hline & Section \#1 & sections \#2 \\
\hline $\mathrm{P}_{\mathrm{in}}, \mathrm{MW}$ & 11 & 11 \\
\hline $\mathrm{I}_{\mathrm{out}}, \mathrm{A}$ & 1,04 & 1,0 \\
\hline $\begin{array}{l}\varepsilon_{\mathrm{x}, \mathrm{y} \text { rms }}, \pi \cdot \mathrm{mm} \cdot \mathrm{mrad} \\
\text { (norm.) }\end{array}$ & 48 & 46 \\
\hline $4 \sigma_{\mathrm{x}, \mathrm{y}}, \mathrm{mm}(\mathrm{B}=0)$ & $8.9(13)$ & $8.8(10,7)$ \\
\hline$\Delta \varphi$ for $70 \%$ of particles & 22 & 21 \\
\hline $\begin{array}{l}\Delta \mathrm{W} / \mathrm{W}, \% \\
\text { for } 70 \% \text { of particles }\end{array}$ & 5 & 3 \\
\hline $\mathrm{W}_{\mathrm{av}}, \mathrm{MeV}$ & 9.4 & 18.9 \\
\hline $\begin{array}{l}\text { beam } \varnothing, \text { mm }(\mathrm{B}=0) \text { for } \\
70 \% \text { of particles }\end{array}$ & $3.6(6)$ & $3.4(5)$ \\
\hline
\end{tabular}

\section{THE LINAC DESIGN}

Structurally an accelerator consists of the radiator and supplying systems. The radiator includes the injector, two accelerating sections, a beam extracting system and a beam parameter monitoring system. The radiator is installed on one stainless steel frame that facilitates mounting the accelerator. The overall length of the radiator is equal $5 \mathrm{~m}$. The beam focussing system has three magnetic axial lenses and short solenoid over the first section. Yokes of the lenses are structural parts of a beam pipe. The vacuum system of the radiator is sealed with metal gaskets. The vacuum is provided with four ion pumps. Extraction of the output beam in atmosphere is implemented through an aluminium foil. For the formation of the necessary irradiation field and for the thermal load lowering on the foil the output beam is scanned in a horizontal plane with frequency of $1-3 \mathrm{~Hz}$ by a scanning magnet. The emission current of the cathode, the beam current at the injector exit and at exits of the first and the second accelerating section are measured with induction current transformers. Besides, at the exit of the second section the induction position monitor of a beam centroid is installed which will be applied also to estimate the beam transverse asymmetry. The general view of the radiator before it has been installed in a shielding tunnel is shown in the fig. 3 . The characteristics of key elements of the radiator are presented in the tables 2 and 3 .

The accelerator is equipped with a RF system, a hard tube electron gun modulator, a cooling system and a power supply system. All systems of the accelerator are controlled by a computerised control system [8]. The RF system includes two powerful amplifying klystrons with modulators and wave-guide system. The part of the output RF power from the klystron that feeds the first accelerating section is divided for the buncher and the accelerating resonator feeding as well as for the excitation of the klystron of the second section by directional couplers and phase shifters. The klystron of the first section operates as a self-excited oscillator. 


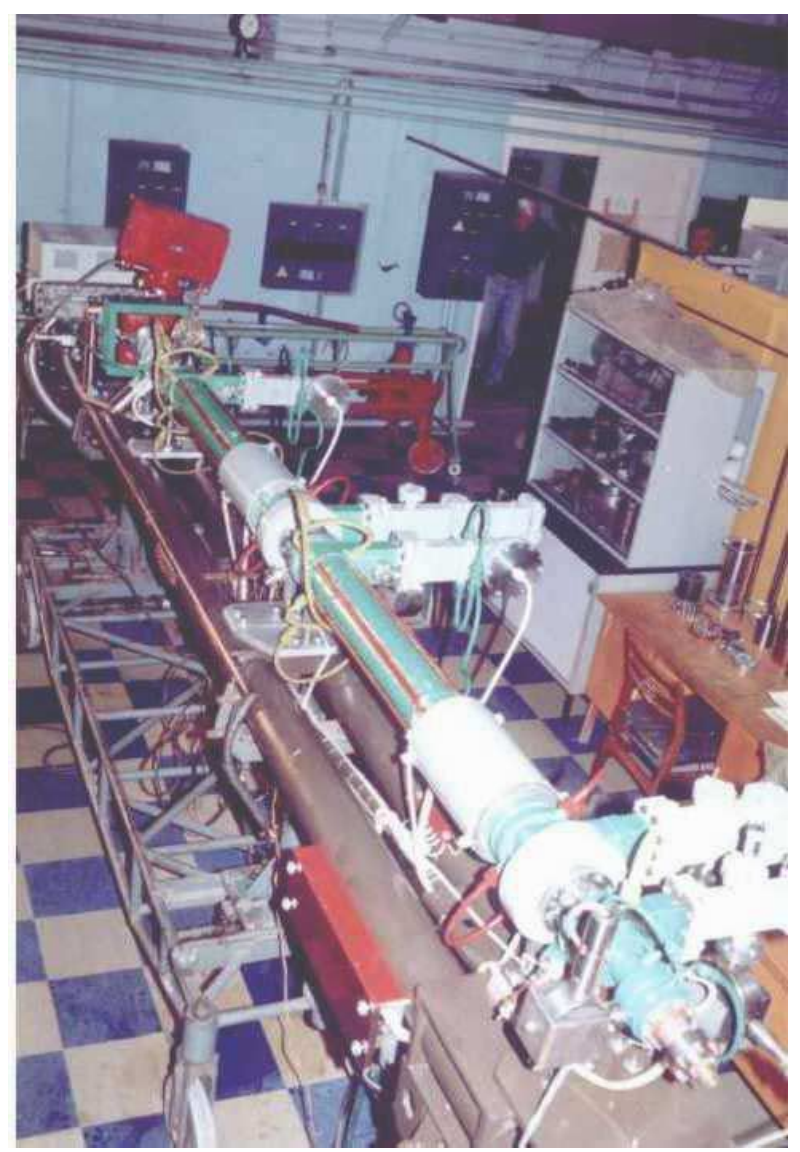

Figure 3: The general view of the radiator.

Table 2. Parameters of buncher and accelerating cavities

\begin{tabular}{|l|l|l|}
\hline & Buncher & Accelerating \\
\hline Type & Reentrant E010 & E010 \\
\hline Frequency, MHz & 2797.2 & 2797.2 \\
\hline Unloaded Q & 3000 & 10000 \\
\hline Coupling & 15 & 5 \\
\hline Shunt imp., M $\Omega$ & 0.39 & 1.1 \\
\hline Input power, MW & 0.0005 & 1.2 \\
\hline $\begin{array}{l}\text { Loaded on-axis } \\
\text { field, MV/m }\end{array}$ & 0.1 & 12 \\
\hline
\end{tabular}

Table 3. Main accelerating section' parameters

\begin{tabular}{|l|l|l|}
\hline & $\mathrm{I}=0 \mathrm{~A}$ & $\mathrm{I}=1.2 \mathrm{~A}$ \\
\hline Frequency, $\mathrm{MHz}$ & 2797.2 & 2797.2 \\
\hline Input power, MW & 13 & 13 \\
\hline Energy gain, MeV & 17.8 & 9.46 \\
\hline Accelerating gradient, MeV/m & 14.3 & 7.57 \\
\hline Section length, $\mathrm{m}$ & 1.2 & 1.2 \\
\hline Filling time, $\mu \mathrm{s}$ & 0.31 & 0.31 \\
\hline Field attenuation, Nep/sect. & 0.24 & 4.1 \\
\hline Output power, MW & 8 & 0.035 \\
\hline Numb. of homogenous landings & 4 & 4 \\
\hline
\end{tabular}

The basic performances of klystron modulators and performances of klystrons are presented in the tables 4 and 5 accordingly.

Table 4. Specification of the klystron modulators

\begin{tabular}{|l|l|}
\hline Peak power, MW & 55 \\
\hline Output pulse voltage, kV & 250 \\
\hline
\end{tabular}

\begin{tabular}{|l|l|}
\hline Pulse transformer ratio & $1: 15.5$ \\
\hline Repetition rate, pps & 300 \\
\hline Pulse length, $\mu \mathrm{s}$ & 4.6 \\
\hline Pulse rise time, $\mu \mathrm{s}$ & 0.5 \\
\hline Pulse fall time, $\mu \mathrm{s}$ & 0.7 \\
\hline Pulse top flatness, $\%$ & 1 \\
\hline PFN impedance, $\Omega$ & 8 \\
\hline Thyratron current, kA & 4 \\
\hline
\end{tabular}

Table 5. Specification of the klystrons

\begin{tabular}{|l|l|}
\hline Frequency, $\mathrm{MHz}$ & 2797.2 \\
\hline Output power, $\mathrm{MW}$ & 15 \\
\hline Pulse length, $\mu \mathrm{s}$ & 4.2 \\
\hline Repetition rate, pps & 300 \\
\hline Gain, dB & 37 \\
\hline Maximum driver power, $\mathrm{kW}$ & 5 \\
\hline Beam voltage, $\mathrm{kV}$ & 240 \\
\hline Beam current, $\mathrm{A}$ & 220 \\
\hline Maximum magnetic field, $\mathrm{kG}$ & 1.1 \\
\hline
\end{tabular}

\section{CONCLUSION}

The fulfilled beam dynamics simulation showed that the accelerating structures get RF focusing. It allows running of the linac even without solenoids over the structures. This feature of the proposed linac increases the reliability of a whole facility and diminishes facility cost. Electron efficiency of the linac is about $80 \%$. According to the simulation the capture ratio in the accelerator will be up to $66 \%$ with average beam power about $20 \mathrm{~kW}$.

At the present time the radiator has been installed and the accelerator supporting systems are assembling and testing.

\section{REFERENCES}

[1] Dovbnya A.N., et. al., "Obtaining power photon beams for medical radionuclides production", VANT, "Nuclear Physics Research" series, 1997, vol 4,5(31,32), p. 154 - 156. (in Russian)

[2] N.I. Aizatsky et. al., "KYT - Industrial Technological Accelerator", Proc. of the 14 Conference on charged particle accelerators, Protvino, 1994, v. 4, p. $259-263$.

[3] Khodak I.V. et. al. "Electron gun for technological linear accelerator", Problems of atomic science and technology, 2000, v. 2 (36), p. 86-88.

[4] W.B. Herrmannsfeldt, "EGUN: Electron Optics Program", Stanford Linear Accelerator Center, SLACPUB-6729, 1994.

[5] L.M. Young. "PARMELA", Los Alamos National Laboratory, LA-UR-96-1835, 1996.

[6] J.H. Billen and L.M. Young. "POISSON/SUPERFISH on PC compatibles", Proc. 1993 Particle Accelerator Conff., Washington (USA), 1993, p. 790-792.

[7] N.I. Aizatsky and L.A. Makhnenko. About current loading of the bunching section of a linear accelerator, GTF, 1982, V. 52, Ser. 4, p. 680-683 (in Russian).

[8] N.I.Aizatsky et. al., "Control system for a linear resonance accelerator of intense electron beams", NIM A352, (1994), pp. $61-62$ 